\title{
Matched $G^{k}$-constructions always yield $C^{k}$-continuous isogeometric elements मे
}

\author{
David Groisser and Jörg Peters * \\ University of Florida, United States
}

\begin{abstract}
$G^{k}$ (geometrically continuous surface) constructions were developed to create surfaces that are smooth also at irregular points where, in a quad-mesh, three or more than four elements come together. Isogeometric elements were developed to unify the representation of geometry and of engineering analysis. We show how matched $G^{k}$ constructions for geometry and analysis automatically yield $C^{k}$ isogeometric elements. This provides a formal framework for the existing and any future isogeometric elements based on geometric continuity.
\end{abstract}

\section{Keywords}

Geometric continuity; Isogeometric element; Extraordinary point; Star point; Irregular point; Isoparametric element

\section{Introduction}

Reparameterization, i.e. change of coordinates, is a key concept underlying both $G^{k}$ constructions of curves and surfaces and isogeometric analysis of physical properties. $G^{k}$ continuity characterizes constructions of $C^{k}$ surfaces such that any two abutting pieces have their derivatives match up to order $k$ after reparameterization (see e.g. Gregory and Hahn, 1987; Boehm, 1988; Peters, 2002). Isogeometric elements are higher-order isoparametric elements of classical engineering analysis (Irons and Zienkiewic, 1968): linear combinations of isogeometric elements serve to approximately compute the solution of a differential equation over bounded, often geometrically non-trivial, regions. The term isogeometric was coined in Hughes et al. (2005) to highlight the case when the region is traced out by (shape) functions drawn from the same space as the problem unknowns (analysis functions, a.k.a. displacement functions). The space is typically spanned by tensor-product splines defined on rectangular domains (de Boor, 2002).

Until (Nguyen et al., 2014), the isogeometric approach has not been investigated near points where more or fewer than four tensor-product elements meet smoothly. (Such points are called irregular, extraordinary or star points.) Earlier work, for example (Wang et al., 2012),

\footnotetext{
This paper has been recommended for acceptance by Rida T. Farouki.

Copyright (C) 2015 Published by Elsevier B.V. All rights reserved.

*Corresponding author. jorg@ cise.ufl.edu (J. Peters)..
} 
has the isogeometric elements join only $C^{0}$ along rays emanating from irregular points.

Since the smooth joining of surface pieces at irregular points is governed by $G^{k}$ relations, it is natural to apply the concept of geometric continuity to constructing everywhere differentiable isogeometric elements. This paper shows that when both the region's parameterization and the analysis functions are drawn from the same space of $G^{k}$ continuous maps then the resulting isogeometric elements are $C^{k}$. This observation formed the background for the second author's presentations in early 2014 Peters (2014), with the publication (Nguyen et al., 2014) illustrating the approach. Already (Kiendl et al., 2009) hinted at the use of geometric continuity by suggesting to uniformly stretch one of the domains of two abutting tensor-product functions. Such stretching represents a very special case of $G^{1}$ continuity, that is called parametric continuity to emphasize that the union of both domains can be embedded in the plane by an affine change of variables. However, uniform stretching does not provide the change of variables necessary to address the general challenge presented by irregular points.

The goal of the present paper is to formally prove the statement of the title and to thereby provide a theoretical framework for $G^{k}$-based $C^{k}$-elements. Such elements can readily be constructed from the rich literature on geometric surface constructions or be based on new constructions in that field. For example, the construction in Scott et al. (2013) (see also Westgaard and Nowacki, 2001; Peters, 1995) could be used to build an isogeometric element rather than to just serve as a surface definition in the context of the boundary element method (there, linear combinations of trivariate Green's functions are to be determined, a setup quite different from the one discussed in the present paper).

To keep the paper focused, we do not discuss the interesting and practically relevant issue of polynomial reproduction near irregular points, a property known under the name of 'flexibility' in the geometric design literature. It is only recently that $G^{2}$ surface constructions that reproduce all quadratic modes at the extraordinary point have been derived (see e.g. Karčiauskas and Peters, 2015).

The paper also does not comment on the related but different approach of using subdivision surfaces for isogeometric analysis (see e.g. Cirak et al., 2002) and it does not comment on methods that approximately enforce angle constraints through penalty functions, such as bending strips (Kiendl et al., 2010).

Most recently, the technical report (Kapl et al., 2014), posted after submission of this paper, follows up on the theory and computations in Peters (2014), Nguyen et al. (2014) by exploring the space of $G^{1}$ functions.

\section{Jets, geometric continuity and isogeometric elements}

This section reviews the concepts underlying our main observation: jets on a manifold, geometric continuity and the definition of isogeometric elements. 
Jets of order $k$ are a precise way of capturing "all information of order $\leq k$ at a point." The $k$ jet of an $\mathbb{R}^{d}$-valued $C^{k}$ map defined on an open neighborhood of a point s $\in \mathbb{R}^{m}$ is an equivalence class defined as follows. Fix an integer $k \geq 1$. Given $m, d \geq 1 \geq$ and a point $\mathrm{s} \in \mathbb{R}^{m}$, consider the set of pairs

$\mathscr{F}_{\mathrm{s}, d}:=\left\{(f, \mathscr{N}) \mid \mathscr{N} \quad\right.$ is an $\quad \mathbb{R}^{m}$ - open neighborhood of $\mathrm{s}$ and $f: \mathscr{N} \rightarrow \mathbb{R}^{d}$ is $\left.\quad C^{k}\right\}$.

For each $m$-tuple $\stackrel{\circ}{\square}:=\left(i_{1}, \ldots, i_{m}\right)$, where the $i_{j}$ are non-negative define $|\stackrel{\circ}{\square}|=\sum i_{j}$ and let $\partial_{\square}$ denote the $\mid \stackrel{\circ}{\square}$ th-order partial-differentiation $\left(\frac{\partial}{\partial x_{1}}\right)^{i_{1}} \ldots\left(\frac{\partial}{\partial x_{m}}\right)^{i_{m}}$. The relation $\sim_{\mathrm{s}}^{k}$ on $F_{s, d}$ defined by

$$
\left(f_{1}, \mathscr{N}_{1}\right) \sim_{\mathrm{s}}^{k}\left(f_{2}, \mathscr{N}_{2}\right) \quad \text { if } \quad \partial_{\circ} f_{1}(\mathrm{~s})=\partial_{\circ} f_{2}(\mathrm{~s}) \quad \text { for } \text { all } \stackrel{\circ}{\square} \text { with }|\stackrel{\circ}{\square}| \leq k,
$$

is an equivalence relation, and the equivalence class of $f$ under $\sim_{\mathrm{s}}^{k}$ is the $k$-jet of fat $s$, denoted $\mathbf{j}_{\mathrm{s}}^{k} f$. We write $J_{\mathrm{s}, d}^{k}$ for the set of $k$-jets of $\mathbb{R}^{d}$-valued maps at s. Note that if $\left(f_{1}, \mathscr{N}_{1}\right) \sim_{\mathrm{s}}^{k}\left(f_{2}, \mathscr{N}_{2}\right)$ then, taking $|\stackrel{\circ}{\square}|=0$ in $(2)$, we have $f_{1}(s) f_{2}(s)$.

If $U \subset \mathbb{R}^{m}, V \subset \mathbb{R}^{\ell}$ are open, we can consider composable $C^{k}$ maps: $f: U \rightarrow V, g: V \rightarrow \mathbb{R}^{d}$. Using the chain rule it is easily seen that

$$
\begin{gathered}
\text { if }\left(f_{1}, U_{1}\right) \sim_{\mathrm{s}}^{k}\left(k_{2}, U_{2}\right) \text { and }\left(g_{1}, V_{1}\right) \sim_{f(\mathrm{~s})}^{k}\left(g_{2}, V_{2}\right), \\
\text { then }\left(g_{1} \circ f_{1}, U_{1}\right) \sim_{\mathrm{s}}^{k}\left(g_{2} \circ f_{2}, U_{2}\right),
\end{gathered}
$$

where $f(s):=f_{l}(s)=f_{2}(s)$ and ${ }^{\circ}$ denotes composition. This implies that there is a well-defined composition map $\circ: J_{f(\mathrm{~s}), d}^{k} \times J_{\mathrm{s}, \ell}^{k} \rightarrow J_{\mathrm{s}, d}^{k}$, dsatisfying

$$
\mathbf{j}_{\mathrm{s}}^{k}(g \circ f)=\left(\mathbf{j}_{f(\mathrm{~s})}^{k} g\right) \circ\left(\mathbf{j}_{\mathrm{s}}^{k} f\right) .
$$

In particular, this applies to the case in which $l=m$ and $g$ is a $C^{k}$ local diffeomorphism. This implies that jets and jet composition are well-defined when $\mathbb{R}^{m}, \mathbb{R}^{\ell}, \mathbb{R}^{d}$ are replaced by $C^{k}$ manifolds. That is, $k$-jets of maps from one $C^{k}$ manifold to another are well-defined, and the composition law (3) holds in this larger context.

Appendix A shows that the definition of jets also carries over, essentially unchanged, to half-spaces. Jets on half-spaces are used in the definition of geometric continuity below.

\section{Geometric \\ Continuity}

Geometric continuity refers to matching geometric invariants. However, for practical constructions, the following parameterization-based definition of matching derivatives after 
a change of coordinates is widely accepted and equivalent in most relevant cases (Peters, 2002, Sect. 3). We formulate geometric continuity in $m$ variables as follows.

Fix $k \geq 1$. For $i=1,2$, let $\square_{i} \subset \mathbb{R}^{m}$ be an $m$-dimensional polytope, for example a unit cube, and let $E_{i}$ be an $(m-1)$-dimensional facet of ${ }_{\square_{i}}$, with interior $\operatorname{int}\left(E_{i}\right)=E\left(m_{i}\right)=E_{i}$. (Here and below, when we refer to the interior $\stackrel{\circ}{P}$ of a $j$-dimensional polytope $P$, we mean the interior of $P$ as a $j$-dimensional object.) Suppose that we are given open sets $\mathscr{N}_{1}, \mathscr{N}_{2} \subset \mathbb{R}^{m}$ containing $E_{1}, E_{2}$ respectively, and a $C^{k}$ diffeomorphism

$$
\begin{aligned}
& \left.\rho: \mathscr{N}_{1} \rightarrow \mathscr{N}_{2} \quad \text { such that (cf.Fig. } 1\right) \\
& \rho\left(\stackrel{\circ}{E}_{1}\right)=\stackrel{\circ}{E}_{2}, \quad \rho\left(\mathscr{N}_{1} \cap \stackrel{\circ}{\square}_{1}\right)=\mathscr{N}_{2} \backslash \square_{2}, \quad \rho\left(\mathscr{N}_{1} \backslash \square_{1}\right)=\mathscr{N}_{2} \cap \stackrel{\circ}{\square}_{2} .
\end{aligned}
$$

Observe that the second line of (4) can equivalently be written as

$$
\begin{gathered}
\rho\left(\stackrel{\circ}{E}_{1}\right)=\stackrel{\circ}{E_{2}} m, \quad \rho\left(\mathscr{N}_{1} \cap \square_{1}\right)=\mathscr{N}_{2} \backslash \stackrel{\circ}{\square_{2}}, \quad \rho\left(\mathscr{N}_{1} \backslash \stackrel{\circ}{\square}\right)=\mathscr{N}_{2} \cap \square_{2} \\
\mathbf{x}_{2}(\rho(\mathrm{s}))=\mathbf{x}_{1}(\mathrm{~s}) \quad \text { for all } \quad \mathrm{s} \in \stackrel{\circ}{E}_{1} ; \quad \text { (6) }
\end{gathered}
$$

thus the images of the $\mathbf{x}_{i}$ join along a common interface $E:=\mathbf{x}_{2}\left(E_{2}\right)=\mathbf{x}_{1}\left(E_{1}\right)$. We say that $\mathbf{x}_{1}$ joins $\mathbf{x}_{2} G^{k}$ with reparameteri-zation $\rho$ along $E$ if for every $\in_{\mathrm{s}} \in \stackrel{\circ}{E}_{1}$ we have

$$
\mathbf{j}_{\mathrm{s}}^{k} \mathbf{x}_{1}=\mathbf{j}_{\mathrm{s}}^{k}\left(\mathbf{x}_{2} \circ \rho\right)
$$

When $m=2, d=3$, and $\mathbf{x}_{\mathrm{i}}$ are tensor-product splines then each $\square_{i}$ is a rectangle and $E$ is a boundary curve shared by the When $m=2, d=3$, and $\mathbf{x}_{i}$ are tensor-product splines then each ${ }_{i}$ surface pieces $\mathbf{x}_{1}\left(\square_{1}\right)$ and $\mathbf{x}_{2}\left(\square_{2}\right)$ (see Fig. 1). Such pairwise $G^{k}$ constructions are used to assemble surfaces where three or more than four tensor-product splines are to be joined smoothly to enclose a point; placing the rectangular domains directly, without reparameterization, into $\mathbf{R}^{2}$ to form a joint domain yields an embedding only if exactly four rectangles meet.

The maps $\mathbf{x}_{i}$ with properties (6) and (7) will be used to provide a manifold domain. More generally, if functions $u_{i}: \square_{i} \rightarrow \mathbb{R}^{N}$ satisfy $\mathbf{j}_{\mathrm{s}}^{k}\left(u_{1}\right)=\mathbf{j}_{\mathrm{s}}^{k}\left(u_{2} \circ \rho\right)$ for all $\mathrm{s} \in \stackrel{\circ}{E_{1}}$, we say that $u_{1}$ joins $u_{2} G^{k}$ with reparameterization $\rho$.

\section{Isogeometric elements}

In the isoparametric approach to solving partial differential equations, maps $\mathbf{x}_{i}, i=1,2, \ldots, n$, parameterize a region or manifold $X$ called the physical domain. The maps $\mathbf{x}_{i}$ are typically splines that map into $\mathbb{R}^{d}$, i.e. with $d=2$ or $d=3$ component functions. The physical domain is tessellated into pieces $\mathbf{x}_{i}\left(\square_{i}\right)$,

$$
X:=\cup_{i=1}^{n} \mathbf{x}_{i}\left(\square_{i}\right) \subset \mathbb{R}^{d}, \quad \mathbf{x}_{i}: \square_{i} \subset \mathbb{R}^{m} \rightarrow \mathbb{R}^{d}
$$


In the following we assume that each $\mathbf{x}_{i}$ is injective on its domain; hence $\mathbf{x}_{i}^{-1}$ is a welldefined map from the image of $\mathbf{x}_{i}$ to $\square_{i}$.

When $m=2$, i.e. in two variables, the $\square_{i}$ may be, for example, unit squares. If $m=2$ and $d=$ 2 then $X$ is a region of the $x y$-plane. If $m=2$ and $d=3, X$ is a surface embedded in $\mathbb{R}^{3}$. If $m$ $=d=3$ then $X$ is a (solid) region in $\mathbb{R}^{3}$.

The goal of the isoparametric approach is to compute, for some $N$, analysis functions $u_{i}: \square_{i}$ $\rightarrow R^{N}$ such that $u_{i} \circ \mathbf{x}_{i}^{-1}$ isolves a partial differential equation on $\mathbf{x}_{i}\left(\square_{i}\right)$. The composition uiox-1iis called (a piece ${ }^{1}$ of) an isogeometric element if the scalar component functions of both $u_{i}$ and $\mathbf{x}_{i}$ are drawn from the same function-space, typically a space of tensor-product spline functions on $\square_{i}$.

In the following, we can confine our attention to two maps, i.e. $i=1,2(n=2)$.

\section{Smoothness of the composition}

We want to show that $G^{k}$ constructions yield $C^{k}$ isogeometric elements. The proof is an adaptation of the definition of a smooth function on a manifold. First we identify a $C^{k}$ atlas on a domain $X_{12}$ defined by joining $\mathbf{x}_{1}$ and $\mathbf{x}_{2}$ with $G^{k}$ continuity. With this atlas, $X_{12}$ becomes a $C^{k}$ manifold as we will now explain in detail.

$C^{k}$ atlas from $G^{k}$ continuity. Consider injective maps $\mathbf{x}_{1}, \mathbf{x}_{2}, \rho$ satisfying the assumptions of (4) and (7), and such that the images $\mathbf{x}_{1}\left(\stackrel{\circ}{\square_{1}}\right), \mathbf{x}_{2}\left(\stackrel{\circ}{\square_{2}}\right)$ Define $\stackrel{\circ}{E}=\mathbf{x}_{1}\left(\stackrel{\circ}{E_{1}}\right)\left(=\mathbf{x}_{2}\left(\stackrel{\circ}{E_{2}}\right)\right.$ by hypothesis). We exhibit a $C^{k}$-manifold structure on the set

$$
\stackrel{\circ}{X_{12}}:=\operatorname{int}\left(\mathbf{x}_{1}\left(\square_{1}\right) \cup \mathbf{X}_{2}\left(\square_{2}\right)\right):=\mathbf{x}_{1}\left(\stackrel{\circ}{\square_{1}}\right) \cup \stackrel{\circ}{E} \cup \mathbf{x}_{2}\left(\stackrel{\circ}{\square_{2}}\right)
$$

as follows. With $N_{i}$ an open set containing $\stackrel{\circ}{E_{1}}, V_{i}:+\stackrel{\circ}{\square}_{i} \cup \mathscr{N}_{i}$ is an extension of the interior of the domain $\square_{i}$. We extend $\left.\mathbf{x}_{1}\right|_{\square_{1}} ^{\circ}$ to a function

$$
\tilde{\mathbf{x}}_{1}: V_{1} \rightarrow \mathbb{R}^{d}, \quad \tilde{\mathbf{x}}_{1}(\mathrm{~s}):=\left\{\begin{array}{lll}
\mathbf{x}_{1}(\mathrm{~s}) & \text { if } & \mathrm{s} \in V_{1} \cap \square_{1}, \\
\mathbf{x}_{2}(\rho(\mathrm{s})) & \text { if } & \mathrm{s} \in V_{1} \backslash \square_{1} \backslash \mathscr{N}_{1}=\square_{1}
\end{array}\right.
$$

and extend $\left.\mathbf{x}_{2}\right|_{\square_{2}} ^{\circ}$ to an analogous function

$$
\tilde{\mathbf{x}}_{2}: V_{2} \rightarrow \mathbb{R}^{d}, \quad \tilde{\mathbf{x}}_{2}(\mathrm{~s}):= \begin{cases}\mathrm{x}_{2}(s) & \text { if } \quad \mathrm{s} \in V_{2} \cap \square_{2}, \\ \mathbf{x}_{1}\left(\rho^{-1}(\mathrm{~s})\right) & \text { if } \quad \mathrm{s} \in V_{2}=\square_{2} \backslash \mathscr{N}_{2} \backslash \square_{2},\end{cases}
$$

\footnotetext{
${ }^{1}$ Iso-geometric degrees of freedom are typically associated with a union of pieces $\mathbf{x}_{i}(\square i)$ of the physical domain (8). (see e.g. Nguyen et al., 2014).
} 


$$
=\left\{\begin{array}{lll}
\mathbf{x}_{2}(\mathrm{~s}) & \text { if } & \mathrm{s} \in V_{2} \cap \stackrel{\circ}{\square}_{2} \\
\mathbf{x}_{2}\left(\rho^{-1}(\mathrm{~s})\right) & \text { if } & \mathrm{s} \in V_{2} \backslash \stackrel{\circ}{\square}_{2} \backslash \mathscr{N}_{2}=\stackrel{\circ}{\square}_{2}
\end{array}\right.
$$

(The equality between (11) and (12) follows from (6).) Because of the jet-equality (7), the extended maps $\tilde{\mathbf{x}}_{i}$ are $C^{k}$. Regarding $\tilde{\mathbf{x}}_{i}$ as a map from $V_{i}$ to $\tilde{\mathbf{x}}_{i}\left(V_{i}\right)$, we write $\tilde{\mathbf{x}}_{i}^{-1}$ for the inverse of this map. Now observe that (12) and (5) imply that

$$
\tilde{\mathbf{x}}_{2}^{-1}(\mathbf{y})=\left\{\begin{array}{lll}
\mathbf{x}_{2}^{-1}(\mathbf{y}) & \text { if } & \mathbf{y} \in \mathbf{x}_{2}\left(V_{2} \cap \stackrel{\circ}{\square}_{2}\right) \\
\rho\left(\mathbf{x}_{1}^{-1}(\mathbf{y})\right) & \text { if } & \mathbf{y} \in \mathbf{x}_{1}\left(\rho^{-1}\left(\mathscr{N}_{2} \backslash \circ_{2}\right)\right)=\mathbf{x}_{1}\left(\mathscr{N}_{1} \cap \square_{1}\right) .
\end{array}\right.
$$

If $\mathrm{s} \in N_{1} \square_{1}$ then $\tilde{\mathbf{x}}_{1}(\mathrm{~s}) \in \mathbf{x}_{2}\left(\rho\left(\mathscr{N}_{1} \backslash \square_{1}\right)\right)=\mathbf{x}_{2}\left(\mathscr{N}_{2} \cap \stackrel{\circ}{\square}_{2}\right) \subset \mathbf{x}_{2}\left(V_{2} \cap \stackrel{\circ}{\square_{2}}\right)$, implying $\tilde{\mathbf{x}}_{2}^{-1}\left(\tilde{\mathbf{x}}_{1}(\mathrm{~s})\right)=\mathbf{x}_{2}^{-1}\left(\mathbf{x}_{2}(\rho(\mathrm{s}))\right)=\rho(\mathrm{s})$, while if $\mathrm{s} \in N_{1} \cap \square_{1}$ then $\left.\tilde{\mathbf{x}}_{1} \mathrm{~s}\right)=\mathbf{x}(\mathrm{s}) \in \mathbf{x}_{1}\left(\mathscr{N}_{1} \cap \square_{1}\right)$, implying $\tilde{\mathbf{x}}_{2}^{-1}\left(\tilde{\mathbf{x}}_{1}(\mathrm{~s})\right)=\rho\left(\mathbf{x}_{1}^{-1}\left(\mathbf{x}_{1}(\mathrm{~s})\right)\right)=\rho(\mathrm{s})$. Hence

$$
\left.\tilde{\mathbf{x}}_{2}^{-1} \circ \tilde{\mathbf{x}}_{1}\right|_{\mathscr{N}_{1}}=\rho \text {, and similarly }\left.\tilde{\mathbf{x}}_{1}^{-1} \circ \tilde{\mathbf{x}}_{2}\right|_{\mathscr{N}_{2}}=\rho^{-1} \text {. }
$$

Therefore $\left\{\left(V_{1}, \tilde{\mathbf{x}}_{1}^{-1}\right),\left(V_{2}, \tilde{\mathbf{x}}_{2}^{-1}\right)\right\}$ is a $C^{k}$ atlas on $\stackrel{\circ}{X_{12}}$ as claimed.

This observation generalizes and makes more precise a similar statement for surfaces in Peters (2002, Sect. 3.2).

\section{The main result}

We are now ready to prove the main result.

\section{Theorem 1}

(Matched $\mathrm{G}^{\mathrm{k}}$ constructions yield $\mathrm{C}^{\mathrm{k}}$ isogeometric elements). For $\mathrm{i}=1,2$, consider $\mathrm{C}^{\mathrm{k}}$ maps $u_{i}: \square_{i} \rightarrow \mathbb{R}^{N}$ and $\mathbf{x}_{i}: \square_{i} \rightarrow \mathbb{R}^{d}$ and assume that each $\mathbf{x}_{\mathbf{i}}$ is injective and the images $\mathbf{x}_{1}\left(\square^{\circ}\right)$, $\mathbf{x}_{2}\left({\left.\stackrel{\circ}{\square_{2}}\right)}_{2}\right.$ are disjoint. Assume that $\mathbf{x}_{1}$ joins $\mathbf{x}_{2} \mathrm{G}^{\mathrm{k}}$ along $\mathrm{E}:=\mathbf{x}_{1}\left(\mathrm{E}_{1}\right)=\mathbf{x}_{2}\left(\mathrm{E}_{2}\right)$ with reparameterization $\rho$ (defined in (4)) and that the analysis functions uimatch the setup in that $\mathrm{u}_{1}$ joins $\mathrm{u}_{2} \mathrm{G}^{\mathrm{k}}$ with the same reparameterization $\rho$. Let $\tilde{u}_{12}: \stackrel{\circ}{X}_{12} \rightarrow \mathbb{R}^{N}$ (with $\stackrel{\circ}{12}_{1}$ defined in (9)) be the piecewise isogeometric element defined by

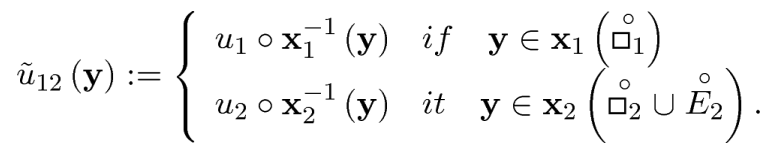

Then

- $\tilde{u}_{12}$ is $C^{k}$ and 
- $\tilde{u}_{12}$ is the unique function $\stackrel{\circ}{12}_{1} \rightarrow \mathbb{R}^{N}$ that restricts to $u_{i} \circ \mathbf{x}_{i}^{-1}$ on

$$
\mathbf{x}\left(\stackrel{\circ}{\square}_{1} \cup \stackrel{\circ}{E}_{i}\right) \quad \text { for } \quad i=1,2 \text {. }
$$

\section{Proof}

The $G^{k}$-join condition on the $u_{i}$ ensures that

$$
u_{2}(\rho(\mathrm{s}))=u_{1}(\mathrm{~s}) \quad \text { for all } \mathrm{s} \in \stackrel{\circ}{E}_{1}, \quad(16)
$$

which together with the $G^{k}$-join condition on the $\mathbf{x}_{i}$ implies that

$$
u_{1} \circ \mathbf{x}_{1}^{-1}(\mathbf{y})=u_{2} \circ \mathbf{x}_{2}^{-1} \quad \text { for all } \mathbf{y} \in \stackrel{\circ}{E}:=\mathbf{x}_{1}\left(\stackrel{\circ}{E_{1}}\right)=\mathbf{x}_{2}\left(\stackrel{\circ}{E_{2}}\right) .
$$

Hence $\tilde{u}_{12}$ agress with $u_{1} \circ \mathbf{x}_{1}^{-1}$ on $\mathbf{x}_{1}\left(\stackrel{\circ}{E_{1}}\right)$ as well as on $\mathbf{x}_{1}\left(\stackrel{\circ}{\square_{1}}\right)$. Therefore $\tilde{u}_{12}$ agrees with $u_{i} \circ \mathbf{x}_{i}^{-1}$ on $\mathbf{x}_{i}\left(\stackrel{\circ}{\square_{i}} \cup \stackrel{\circ}{E}_{i}\right)$ for both $i=1$ and $i=2$. Uniqueness of a function $\stackrel{\circ}{12}_{12} \rightarrow \mathbb{R}^{N}$ that restricts to $u_{i} \circ \mathbf{x}_{i}^{-1}$ on $\mathbf{x}_{i}\left(\stackrel{\circ}{\square}_{i} \cup \stackrel{\circ}{E_{i}}\right)$ for both $i$ is clear, since any such function satisfies the defining equation (15) for $\tilde{u}_{12}$.

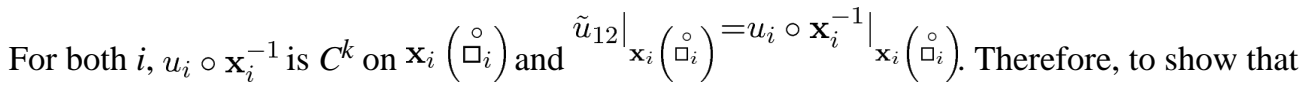
$\tilde{u}_{12}$ is $C^{k}$, it suffices to verify that $\tilde{u}_{12}$ is $C^{k}$ at each point of ${ }_{E}^{\circ}$. Hence it succes to check that $\mathbf{j}_{\mathbf{y}}^{k}\left(u_{2} \circ \mathbf{x}_{2}^{-1}\right)=\mathbf{j}_{\mathbf{y}}^{k}\left(u_{1} \circ \mathbf{x}_{1}^{-1}\right)$ at every point $\mathbf{y} \in \stackrel{\circ}{E}$.

Define $\tilde{\mathbf{x}}_{1}, \tilde{\mathbf{x}}_{2}$ as in (10)and (11). The assumed injectivity of the $\mathbf{x}_{i}$ and disjointness of the images $\mathbf{x}_{1}\left(\stackrel{\circ}{1}_{1}\right), \mathbf{x}_{2}\left(\stackrel{\circ}{\square}_{2}\right)$ imply that (14) holds; in particular, that $\left.\tilde{\mathbf{x}}_{2}^{-1}\right|_{\tilde{\mathbf{x}}_{1}\left(\mathscr{N}_{1}\right)}=\left.\rho \circ \tilde{\mathbf{x}}_{1}^{-1}\right|_{\tilde{\mathbf{x}}_{1}\left(\mathscr{N}_{1}\right)}$. Applying the composition law (3) to $C^{k}$ maps between $C^{k}$ manifolds-with-boundary and omitting the notation indicating restriction to $\tilde{\mathbf{x}}_{1}\left(\mathscr{N}_{1}\right)$, for all $\mathbf{y} \in \stackrel{\circ}{E}$ (a subnet of $\left.\tilde{\mathbf{x}}_{1}\left(\mathscr{N}_{1}\right)\right)$, , we have

$$
\begin{aligned}
\mathbf{j}_{\mathbf{y}}^{k}\left(u_{2} \circ \mathbf{x}_{2}^{-1}\right) & =\mathbf{j}_{\mathbf{y}}^{k}\left(u_{2} \circ \tilde{x}_{2}^{-1}\right) \\
& =\mathbf{j}_{\mathbf{y}}^{k}\left(u_{2} \circ \rho \circ \tilde{x}_{1}^{-1}\right) \\
& =\left(\mathbf{j}_{\tilde{\mathbf{x}}_{1}^{-1}(\mathbf{y})}^{k}\left(u_{2} \circ \rho\right)\right) \circ \mathbf{j}_{\mathbf{y}}^{k}\left(\tilde{\mathbf{x}}_{1}^{-1}\right) \\
& =\left(\mathbf{j}_{\tilde{\mathbf{x}}_{1}^{-1}(\mathbf{y})}^{k} u_{1}\right) \circ \mathbf{j}_{\mathbf{y}}^{k}\left(\tilde{\mathbf{x}}_{1}^{-1}\right) \\
& =\mathbf{j}_{\mathbf{y}}^{k}\left(u_{1} \circ \tilde{\mathbf{x}}_{1}^{-1}\right)=\mathbf{j}_{\mathbf{y}}^{k}\left(u_{1} \circ \mathbf{x}_{1}^{-1}\right)
\end{aligned}
$$

The first and last equalities above are true by the definition of jets at boundaries of halfspaces; see Appendix A. 
We can extend the $C^{k}$ structure to the outer boundaries of the domains $\square_{i}$ by strengthening the disjointness assumption to $\mathbf{x}_{1}\left(\square_{1}\right) \cap \mathbf{x}_{2}\left(\square_{2}\right)=E$. Indeed, if $Z$ is any metric space and the functions uiare continuous, then (16)is a necessary and sufficient condition for existence and uniqueness of a continuous function $\tilde{u}$ from the larger domain $\mathbf{x}_{1}\left(\square_{1}\right) \cup \mathbf{x}_{2}\left(\square_{2}\right)$ to $Z$ whose restriction to $E$ coincides with both $\left.u_{1} \circ \mathbf{x}_{1}^{-1}\right|_{V_{1}}$ and $\left.u_{2} \circ \mathbf{x}_{2}^{-1}\right|_{V_{2}}$ (where $V_{i}$ are the sets defined prior to (10)). Also, it is not hard to adapt the proof to the case where $E$ is the image of just a piece of the boundary of each $E_{i}$.

\section{Conclusion}

Deriving isogeometric $C^{k}$ elements from $G^{k}$-manifold constructions is natural. The point of Theorem 1 is to formally justify this approach and to lay a foundation from which to address issues of approximation, flexibility and polynomial reproduction. For, as a consequence of Theorem 1, $G^{k}$-manifold constructions can be used to devise smooth isogeometric elements for regular as well as irregular layouts, in any number of variables. In particular, existing $G^{k}$ surface constructions in the literature can directly be used to solve differential equations both on surfaces and on planar regions where more or fewer than four four-sided pieces come together - with the value of each construction depending on their individual approximation and convergence properties.

\section{Acknowledgements}

The work supported in part by the National Science Foundation under grant CCF-1117695 and by the National Institute of Health grant R01-EB018625. The second author thanks B. Mourrain, T. Nguyen and U. Reif for early discussions on the subject.

\section{Appendix A One-sided jets}

We generalize jets by replacing $\mathbb{R}^{m}$ in (1) by a half-space $H$, for example $\mathbb{R}_{+}^{m}:=\mathbb{R}^{m-1} \times[0, \infty)$. A set $U$ is $H$-open if $U=V \cap H$ for some $m$-dimensional open set $V \subset \mathbb{R}^{m}$. Suppose that $U$ is $H$-open and $f: U \rightarrow \mathbb{R}^{\ell}$ is some function on $U$. If s $\in U \partial H$, where $\partial H$ is the boundary of $H$, an ( $m$ 1)-dimensional hyperplane then the definition of " $f$ is $C^{k}$ at s" is the same as if $H$ were replaced by $\mathbb{R}^{\ell}$. If s $\in U \cap \partial H, f$ is $C^{k}$ at s if there exists an extension $\tilde{f}$ of $f$ to some domain that includes an $\mathbb{R}^{m}$-open neighborhood of s such that $\tilde{f}$ is $C^{k}$ at $p$. We can then consider the set

$$
\begin{aligned}
\mathscr{F}_{\mathrm{s}, H, \ell} & :=\{(f, U, \tilde{f}) \mid U \text { is an } H \text {-open neighborhood of } \mathrm{s}, \\
f: U & \rightarrow \mathbb{R}^{\ell} \text { is } C^{k} \text {, and } \tilde{f} \text { is an extension of } U \text { to a domain that includes an } \\
& \left.\mathbb{R}^{m} \text {-open neighborhood of } \mathrm{s}\right\},
\end{aligned}
$$

and the equivalence relation $\sim_{\mathrm{s}}^{\prime}$ defined by

$$
\left(f_{1}, U_{1}, \tilde{f}_{1}\right) \sim_{\mathrm{s}}^{\prime}\left(f_{2}, U_{2}, \tilde{f}_{2}\right) \quad \text { if } \partial_{\square} \tilde{f}_{1}(\mathrm{~s}) \text { for all } \stackrel{\circ}{\square} \text { with }|\stackrel{\circ}{\square}| \leq k .
$$


Denote the set of equivalence classes under this relation by $J_{\mathrm{s}, H, \ell^{*}}^{k}$ There is a natural one-toone correspondence $J_{\mathrm{s}, H, \ell}^{k} \leftrightarrow J_{\mathrm{s}, \ell}^{k}$ given by mapping the equivalence class of $(f, U, \tilde{f})$ under $\sim_{\mathrm{s}}^{\prime}$ to $\mathbf{j}_{\mathrm{s}}^{k} \tilde{f}$. The $k$-jet $\mathbf{j}_{p}^{k} \tilde{f}$ is independent of which $C^{k}$ extension $\tilde{f}$ is chosen, so we can use the notation $\mathbf{j}_{\mathrm{s}}^{k} f$ without confusion. The correspondence $J_{\mathrm{s}, H, \ell}^{k} \leftrightarrow J_{\mathrm{s}, \ell}^{k}$ also makes clear that the composition of $k$-jets of functions defined on half-space domains is well-defined, and that (3) holds in this context as well. It follows that $k$-jets of maps from one $C^{k}$ manifold-withboundary to another are well-defined, and the composition law (3) holds in this setting as well. Consequently we may identify $J_{\mathrm{s}, H, \ell}^{k}$ with $J_{\mathrm{s}, \ell}^{k}$, and dispense with the notation " $J_{\mathrm{s}, H, \ell}^{k}$ ".

\section{References}

Boehm, W. Comput. Aided Des. Vol. 20. Letter to the Editor; 1988. On the definition of geometric continuity.; p. 370-372.

de Boor, CW. Handbook of Computer Aided Geometric Design. Elsevier; 2002. B-spline basics..

Cirak F, Scott MJ, Antonsson EK, Ortiz M, Schröder P. Integrated modeling, finite-element analysis, and engineering design for thin-shell structures using subdivision. Comput. Aided Des. 2002; 34(2): 137-148.

Gregory JA, Hahn JM. Geometric continuity and convex combination patches. Comput. Aided Geom. Des. 1987; 4(1-2):79-89.

Hughes TJR, Cottrell JA, Bazilevs Y. Isogeometric analysis: CAD, finite elements, NURBS, exact geometry and mesh refinement. Comput. Methods Appl. Mech. Eng. 2005; 194:4135-4195.

Irons, BM.; Zienkiewic, OC. Proc. Conf. Recent Advances in Stress Analysis. Royal Aeronautical Society; London: 1968. The isoparametric finite element system - a new concept in finite element analysis..

Kapl, M.; Vitrih, V.; Jüttler, B. Technical Report 22. Joh. Kepler University; 2014. Isogeometric analysis with geometrically continuous functions.; p. G+S

Karčiauskas K, Peters J. Biquintic $\mathrm{G}^{2}$ Surfaces via Functionals. Computer Aided Geometric Design. 2015; $\mathrm{x}-\mathrm{X}+12$ in press.

Kiendl J, Bazilevs Y, Hsu M-C, Wüchner R, Bletzinger K-U. The bending strip method for isogeometric analysis of Kirchhoff-Love shell structures comprised of multiple patches. Comput. Methods Appl. Mech. Eng. 2010; 199:2403-2416.

Kiendl J, Bletzinger K-U, Linhard J, Wüchner R. Isogeometric shell analysis with Kirchhoff-Love elements. Comput. Methods Appl. Mech. Eng. 2009; 198:3902-3914.

Nguyen T, Karčiauskas K, Peters J. A comparative study of several classical, discrete differential and isogeometric methods for solving Poisson's equation on the disk. Axioms. 2014; 3(2):280-299.

Peters J. Biquartic $C^{1}$-surface splines over irregular meshes. Comput. Aided Des. 1995; 27(12):895903.

Peters, J. Handbook of Computer Aided Geometric Design. Elsevier; 2002. Geometric continuity.; p. 193-229.

Peters, J. Isogeometric analysis at irregular points. Presentations at Intl. Workshop on Geometric Design, Schloss Dagstuhl, Germany, May 2014.. Conference on Curves and Surfaces; Paris, France. June 2014; 2014. See also http://arxiv.org/abs/1406.4229

Scott MA, Simpson RN, Evans JA, Lipton S, Bordas SA, Hughes TJR, Sederberg TW. Isogeometric boundary element analysis using unstructured T-splines. Comput. Methods Appl. Mech. Eng. $2013 ; 254: 197-221$.

Wang, Wenyan, Zhang, Yongjie, Xu, Guoliang, Hughes, Thomas J. Converting an unstructured quadrilateral/hexahedral mesh to a rational t-spline. Comput. Mech. 2012; 50(1):65-84.

Westgaard G, Nowacki H. Construction of fair surfaces over irregular meshes. Symposium on Solid Modeling and Applications. 2001:88-98. 


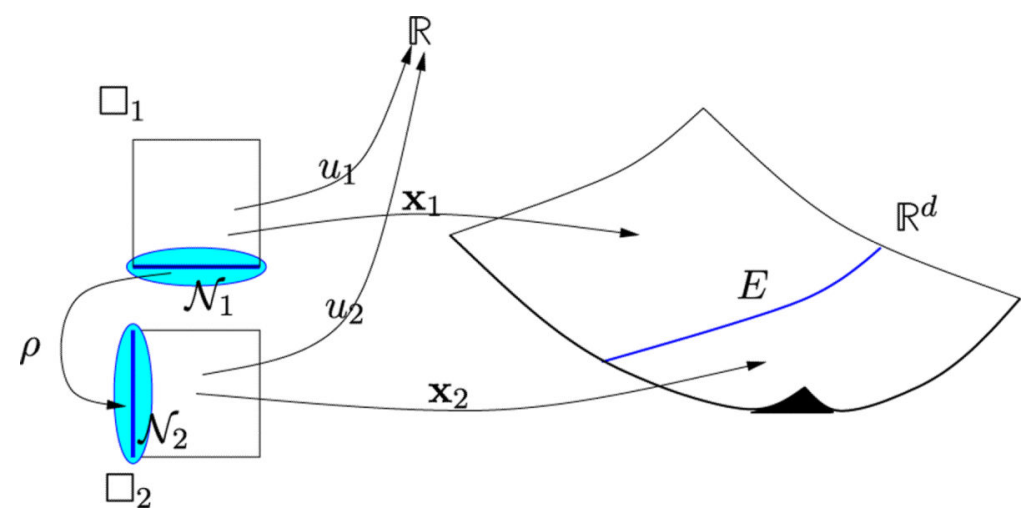

Fig. 1.

Isogeometric elements and $G^{k}$ continuity. 